\title{
Fixed-Point Approximations of Generalized Nonexpansive Mappings via Generalized M-Iteration Process in Hyperbolic Spaces
}

\author{
Preeyalak Chuadchawna, ${ }^{1}$ Ali Farajzadeh, ${ }^{2}$ and Anchalee Kaewcharoen ${ }^{1}{ }^{1}$ \\ ${ }^{1}$ Department of Mathematics, Faculty of Science, Naresuan University, Phitsanulok 65000, Thailand \\ ${ }^{2}$ Department of Mathematics, Razi University, Kermanshah 67149, Iran
}

Correspondence should be addressed to Anchalee Kaewcharoen; anchaleeka@nu.ac.th

Received 5 February 2020; Accepted 7 April 2020; Published 6 May 2020

Academic Editor: Luca Vitagliano

Copyright (c) 2020 Preeyalak Chuadchawna et al. This is an open access article distributed under the Creative Commons Attribution License, which permits unrestricted use, distribution, and reproduction in any medium, provided the original work is properly cited.

In this paper, we propose the generalized M-iteration process for approximating the fixed points from Banach spaces to hyperbolic spaces. Using our new iteration process, we prove $\Delta$-convergence and strong convergence theorems for the class of mappings satisfying the condition $\left(C_{\lambda}\right)$ and the condition $(E)$ which is the generalization of Suzuki generalized nonexpansive mappings in the setting of hyperbolic spaces. Moreover, a numerical example is given to present the capability of our iteration process and the solution of the integral equation is also illustrated using our main result.

\section{Introduction}

The concept of nonexpansive mappings can be defined in many general setting of metric spaces. Suzuki [1] introduced the concept of generalized nonexpansive single-valued mappings which are called Suzuki generalized nonexpansive mappings or the condition $(C)$ and received some fixedpoint results and convergence results for such mappings in Banach spaces. Also, the new conditions for single-valued mappings are defined by García-Falset et al. [2], called the condition $\left(C_{\lambda}\right)$ and the condition $(E)$ which are weaker than a nonexpansive mapping and stronger than a quasi-nonexpansive mapping. Moreover, the class of mappings satisfying the condition $\left(C_{\lambda}\right)$ and the condition $(E)$ is larger than the class of mappings satisfying the condition $(C)$.

Let $T$ be a self-mapping on a nonempty subset $D$ of a Banach space $X$ and $\left\{\omega_{n}\right\}$ and $\left\{\sigma_{n}\right\}$ are real sequences in $[0,1]$ for all $n \geq 0$. Mann [3] iteration process was determined by the following method:

$$
\left\{\begin{array}{l}
x_{0} \in D \\
x_{n+1}=\left(1-\omega_{n}\right) x_{n}+\omega_{n} T x_{n}
\end{array}\right.
$$

The following is called the S-iteration process defined by Agarwal et al. [4]:

$$
\left\{\begin{array}{l}
x_{0} \in D, \\
y_{n}=\left(1-\sigma_{n}\right) x_{n}+\sigma_{n} T x_{n}, \\
x_{n+1}=\left(1-\omega_{n}\right) T x_{n}+\omega_{n} T y_{n} .
\end{array}\right.
$$

Next, Gursoy et al. [5] introduced the Picard-S-iteration process. By providing an example, they presented that Picard-S-iteration process converges faster than all Mann, Picard, Noor, Ishikawa, CR, SP, S, S*, Abbas, and Normal-S involving Two-step Mann iteration processes. The Picard-Siteration process is defined as

$$
\left\{\begin{array}{l}
x_{0} \in D, \\
z_{n}=\left(1-\sigma_{n}\right) x_{n}+\sigma_{n} T x_{n}, \\
y_{n}=\left(1-\omega_{n}\right) T x_{n}+\omega_{n} T z_{n}, \\
x_{n+1}=T y_{n} .
\end{array}\right.
$$

Recently in 2018, Ullah et al. [6] introduced a new iterative process called the $\mathrm{M}$-iteration process defined as follows: 


$$
\left\{\begin{array}{l}
x_{0} \in D, \\
z_{n}=\left(1-\omega_{n}\right) x_{n}+\omega_{n} T x_{n}, \\
y_{n}=T z_{n}, \\
x_{n+1}=T y_{n} .
\end{array}\right.
$$

They proved that $\mathrm{M}$-iteration process can be used to approximate the fixed point of Suzuki generalized nonexpansive mappings and obtain weak convergence and strong convergence results on Banach spaces. They also presented that the $\mathrm{M}$-iteration process converges faster than Picard-S-iteration and S-iteration processes by providing an instance.

Motivated by above, we introduce a generalized M-iteration process and use our iterative scheme for proving some $\Delta$-convergence and strong convergence theorems for mappings satisfying the condition $\left(C_{\lambda}\right)$ and the condition $(E)$ which is the generalization of Suzuki generalized nonexpansive mappings (the condition $(C)$ ) in the setting of hyperbolic spaces. Numerically, we give an example of a mapping satisfying the condition $\left(C_{\lambda}\right)$ and the condition $(E)$, which does not satisfy the condition $(C)$. Finally, we compare the speed of convergence of our generalized M-iteration process with M-iteration process of Ullah et al. [6].

\section{Preliminaries}

For this research, we discuss on the setting of hyperbolic spaces which was introduced by Kohlenbach [7], containing normed linear spaces and convex subsets and Hadamard manifolds [8], CAT(0) spaces in the sense of Gromov [9], and the Hilbert ball equipped with the hyperbolic metric [8].

A hyperbolic space is a triple $(X, d, W)$ where $(X, d)$ is a metric space and $W: X^{2} \times[0,1] \longrightarrow X$ such that

$$
\begin{aligned}
& \text { (W1) } d(w, W(u, v, \omega)) \leq(1-\omega) d(w, u)+\omega d(w, v), \\
& \text { (W2) } d(W(u, v, \omega), W(u, v, \sigma))=|\omega-\sigma| d(u, v), \\
& \text { (W3) } W(u, v, \omega)=W(v, u,(1-\omega)), \\
& (\mathrm{W} 4) d(W(u, z, \omega), W(v, w, \omega)) \leq(1-\omega) d(u, v)+ \\
& \omega d(z, w),
\end{aligned}
$$

for all $u, v, w, z \in X$ and $\omega, \sigma \in[0,1]$.

First we recall some definitions, lemmas, and propositions that will be used in the next part.

A hyperbolic space $(X, d, W)$ is called uniformly convex [10] if for all $u, v, z \in X, r>0$, and $\varepsilon \in(0,2]$ there exists $\delta \in(0,1]$ such that $d(v, u) \leq r, d(z, u) \leq r$, and $d(v, z) \geq \varepsilon r$. Then, we have

$$
d\left(W\left(v, z, \frac{1}{2}\right), u\right) \leq(1-\delta) r .
$$

A mapping $\eta:(0, \infty) \times(0,2] \longrightarrow(0,1]$ which provides $\delta=\eta(r, \varepsilon)$ for a given $r>0$ and $\varepsilon \in(0,2]$ is well known as a modulus of uniform convexity of $X$. We call $\eta$ as a monotone if it decreases with $r$ (for a fixed $\varepsilon$ ), i.e., for any given $\varepsilon>0$ and for any $r_{2} \geq r_{1}>0$, we have $\eta\left(r_{2}, \varepsilon\right) \leq \eta\left(r_{1}, \varepsilon\right)$.
A nonempty subset $D$ of a hyperbolic space $X$ is said to be convex if $W(u, v, \gamma) \in D$ for any $u, v \in D$ and $\gamma \in[0,1]$.

If $u, v \in X$ and $\gamma \in[0,1]$, then we use the notion (1$\gamma) u \oplus \gamma v$ for $W(u, v, \gamma)$. In [10], it is remarked that any normed space $(X,\|\cdot\|)$ is a hyperbolic space, with $(1-\gamma) u \oplus \gamma v:=(1-\gamma) u+\gamma v$. Hence, the class of uniformly convex hyperbolic spaces is a natural generalization of uniformly convex Banach spaces.

Let $D$ be a nonempty subset of metric space $X$. If $T(q)=q$, then $q$ is said to be a fixed point of a mapping $T$. The set of all fixed points of $T$ is denoted by $F(T)$.

Definition 1 (see [11]). A mapping $T: D \longrightarrow D$ is said to be

(i) Nonexpansive if $d(T u, T v) \leq d(u, v)$ for all $u, v \in D$;

(ii) Quasi-nonexpansive if $F(T) \neq \varnothing$ and $d(T u, T q) \leq$ $d(u, q)$ for all $u \in D$ and $q \in F(T)$.

Suzuki [1] defined the notion of Suzuki generalized nonexpansive mappings which is called the condition $(C)$. Such mappings are weaker than nonexpansive mappings and stronger than quasi-nonexpansive mappings.

Definition 2 (see [1]). A mapping $T: D \longrightarrow D$ is called to satisfy the condition $(C)$ if

$$
\frac{1}{2} d(u, T u) \leq d(u, v) \text { implies } d(T u, T v) \leq d(u, v),
$$

for all $u, v \in D$.

Falset et al. [2] introduced the definition of generalized nonexpansive mappings as follows.

Definition 3 (see [2]). A mapping $T: D \longrightarrow D$ is called to satisfy the condition $\left(E_{\mu}\right)$ provided that

$$
d(u, T v) \leq \mu d(u, T u)+d(u, v), \text { for all } u, v \in D .
$$

We say that $T$ satisfies the condition $(E)$ whenever $T$ satisfies the condition $\left(E_{\mu}\right)$ for some $\mu \geq 1$.

Proposition 1 (see [12]). Let $T: D \longrightarrow D$ be a mapping which satisfies the condition $(C)$. Then, $T$ satisfies the condition (E) for some $\mu=3$.

There is an example presented that there exists a mapping which satisfies the condition $(E)$, but it fails to satisfy the condition $(C)$. Then, the mappings satisfying the condition $(E)$ are more generalized than mappings satisfying the condition $(C)$.

Example 1 (see [13]). Let $X=C[0,1]$, and under the supremum norm, consider a nonempty subset $D$ of $X$ defined by

$$
D=\{f \in C[0,1]: 0=f(0) \leq f(1)=1\} .
$$

For any $g \in D$, associate a function $F_{g}: D \longrightarrow D$ defined by $F_{g}(h(t))=\left(g^{\circ} \mathrm{h}\right)(\mathrm{t})=\mathrm{g}(\mathrm{h}(\mathrm{t}))$. It is easy to verify that $F_{g}$ satisfies the condition $\left(E_{1}\right)$ but does not satisfy the condition (C). 
Definition 4 (see [2]). Let $T: D \longrightarrow D$ be a mapping and $\lambda \in(0,1)$. Then, $T$ is said to satisfy the condition $\left(C_{\lambda}\right)$ if for all $u, v \in D$

$$
\lambda d(u, T u) \leq d(u, v) \text { implies } d(T u, T v) \leq d(u, v) .
$$

Of course, it follows that the condition $(C)$ is the special case for $\lambda=1 / 2$ in the condition $\left(C_{\lambda}\right)$.

On the contrary, the notion of a mapping satisfying the condition $I$ was introduced by Senter and Dotson [14].

Definition 5. Let $T: D \longrightarrow D$ said to satisfy the condition $(I)$, if there exists a nondecreasing function $f:[0, \infty) \longrightarrow[0, \infty)$ satisfying $f(0)=0$ and $f(t)>0$ for all $t \in(0, \infty)$, such that

$$
d(v, T v) \geq f(d(v, F(T))) \text { for all } v \in D .
$$

We need the following definition of convergence in hyperbolic spaces [15] which is called $\Delta$-convergence. It plays an essential role in the principle results. In order to define $\Delta$-convergence, we need the following concepts.

Let $\left\{x_{n}\right\}$ be a bounded sequence in a hyperbolic space $X$. We can define a function $r\left(\cdot,\left\{x_{n}\right\}\right): X \longrightarrow[0, \infty)$ by

$$
r\left(x,\left\{x_{n}\right\}\right)=\limsup _{n \rightarrow \infty} d\left(x, x_{n}\right) \text {, for all } x \in X .
$$

An asymptotic radius of a bounded sequence $\left\{x_{n}\right\}$ with respect to a nonempty subset $D$ of $X$ is determined and denoted by

$$
r_{D}\left(\left\{x_{n}\right\}\right)=\inf \left\{r\left(x,\left\{x_{n}\right\}\right): x \in D\right\}
$$

An asymptotic center of a bounded sequence $\left\{x_{n}\right\}$ with respect to a nonempty subset $D$ of $X$ is determined and denoted by

$$
A C_{D}\left(\left\{x_{n}\right\}\right)=\left\{x \in X: r\left(x,\left\{x_{n}\right\}\right) \leq r\left(y,\left\{x_{n}\right\}\right) \text {, for all } y \in D\right\} .
$$

The sequence $\left\{x_{n}\right\}$ in $X$ is said to $\Delta$-converge to $x \in X$ if $x$ is a unique asymptotic center of $\left\{w_{n}\right\}$ for every subsequence $\left\{w_{n}\right\}$ of $\left\{x_{n}\right\}$. In this case, we write $\Delta$ - $\lim _{n \rightarrow \infty} x_{n}=x$ and call $x$ the $\Delta$-lim of $\left\{x_{n}\right\}$.

Next, we review some definitions and lemmas.

Lemma 1 (see [16]). Let $X$ be a complete uniformly convex hyperbolic space with a monotone modulus of uniform convexity $\eta$. Then, every bounded sequence $\left\{x_{n}\right\}$ in $X$ has a unique asymptotic center with respect to any nonempty closed convex subset $D$ of $X$.

Lemma 2 (see [17]). Let $X$ be a complete uniformly convex hyperbolic space with a monotone modulus of uniform convexity $\eta$. Let $x \in X$ and $\left\{\alpha_{n}\right\}$ be a sequence in $[a, b]$ for some $a, b \in(0,1)$. If $\left\{x_{n}\right\}$ and $\left\{y_{n}\right\}$ are sequences in $X$ such that $\lim \sup _{n \rightarrow \infty} d\left(x_{n}, x\right) \leq c$, $\limsup _{n \rightarrow \infty} d\left(y_{n}, x\right) \leq c$, and $\lim _{n \longrightarrow \infty} d\left(W\left(x_{n}, y_{n}, \alpha_{n}\right), x\right)=c$ for some $c \geq 0$. Then,

$$
\lim _{n \longrightarrow \infty} d\left(x_{n}, y_{n}\right)=0
$$

Lemma 3 (see [18]). Let $D$ be a nonempty closed convex subset of a complete uniformly convex hyperbolic space $X$ with a monotone modulus of uniform convexity $\eta$. Let $T: D \longrightarrow D$ be a mapping which satisfies the condition $\left(C_{\lambda}\right)$ for some $\lambda \in(0,1)$ and the condition (E). Suppose that $\left\{x_{n}\right\}$ is a bounded sequence in $D$ such that $\lim _{n \longrightarrow \infty} d\left(x_{n}, T x_{n}\right)=0$. Then, $T$ has a fixed point.

As a consequence of Theorem 3.2 [18], we receive the following lemma.

Lemma 4. Let $D$ be a nonempty closed convex subset of a complete uniformly convex hyperbolic space $X$ with a monotone modulus of uniform convexity $\eta$. Let $T: D \longrightarrow D$ be a mapping which satisfies the condition $\left(C_{\lambda}\right)$ for some $\lambda \in(0,1)$. Then, $F(T)$ is closed.

\section{Main Results}

For this section, we construct an iteration process in hyperbolic spaces named as "generalized M-iteration process," as follows:

$$
\left\{\begin{array}{l}
x_{0} \in D, \\
z_{n}=W\left(x_{n}, T x_{n}, \omega_{n}\right), \\
y_{n}=W\left(T z_{n}, z_{n}, \sigma_{n}\right), \\
x_{n+1}=W\left(T y_{n}, y_{n}, \gamma_{n}\right),
\end{array}\right.
$$

for all $n \geq 0,\left\{\omega_{n}\right\},\left\{\sigma_{n}\right\}$, and $\left\{\gamma_{n}\right\}$ are real sequences in $[0,1]$.

Remark that if we take $\sigma_{n}=\gamma_{n}=0$, then we obtain that (15) reduces to (4).

The following lemmas play crucial role in proving the main theorems of this section.

Throughout in this paper, let $(X, d, W)$ be a complete uniformly convex hyperbolic space with a monotone modulus of uniform convexity $\eta$ and $D$ be a nonempty closed convex subset of $X$.

Lemma 5. Let $D$ be a nonempty closed convex subset of $X$ and $T: D \longrightarrow D$ be a mapping which satisfies the condition $\left(C_{\lambda}\right)$ for some $\lambda \in(0,1)$ with $F(T) \neq \varnothing$. For an arbitrary chosen $x_{0} \in D$, a sequence $\left\{x_{n}\right\}$ is defined by (15). Then, $\lim _{n \longrightarrow \infty} d\left(x_{n}, q\right)$ exists for each $q \in F(T)$.

Proof. Assume that $q \in F(T)$ and $z \in D$. By hypothesis, we obtain that $T$ satisfies the condition $\left(C_{\lambda}\right)$ for some $\lambda \in(0,1)$. So

$\lambda d(q, T q)=0 \leq d(q, z)$ implies that $d(T q, T z) \leq d(q, z)$.

Thus using (15), we obtain that

$$
\begin{aligned}
d\left(z_{n}, q\right) & =d\left(W\left(x_{n}, T x_{n}, \omega_{n}\right), q\right) \\
& \leq\left(1-\omega_{n}\right) d\left(x_{n}, q\right)+\omega_{n} d\left(T x_{n}, q\right) \\
& =\left(1-\omega_{n}\right) d\left(x_{n}, q\right)+\omega_{n} d\left(T x_{n}, T q\right) \\
& \leq\left(1-\omega_{n}\right) d\left(x_{n}, q\right)+\omega_{n} d\left(x_{n}, q\right) \\
& \leq d\left(x_{n}, q\right) .
\end{aligned}
$$


Using (15) together with (17), we get

$$
\begin{aligned}
d\left(y_{n}, q\right) & =d\left(W\left(T z_{n}, z_{n}, \sigma_{n}\right), q\right) \\
& \leq\left(1-\sigma_{n}\right) d\left(T z_{n}, q\right)+\sigma_{n} d\left(z_{n}, q\right) \\
& =\left(1-\sigma_{n}\right) d\left(T z_{n}, T q\right)+\sigma_{n} d\left(z_{n}, q\right) \\
& \leq\left(1-\sigma_{n}\right) d\left(z_{n}, q\right)+\sigma_{n} d\left(z_{n}, q\right) \\
& \leq d\left(z_{n}, q\right) \\
& \leq d\left(x_{n}, q\right) .
\end{aligned}
$$

Again, using (15) and (18), we obtain that

$$
\begin{aligned}
d\left(x_{n+1}, q\right) & =d\left(W\left(T y_{n}, y_{n}, \gamma_{n}\right), q\right) \\
& \leq\left(1-\gamma_{n}\right) d\left(T y_{n}, q\right)+\gamma_{n} d\left(y_{n}, q\right) \\
& =\left(1-\gamma_{n}\right) d\left(T y_{n}, T q\right)+\gamma_{n} d\left(y_{n}, q\right) \\
& \leq\left(1-\gamma_{n}\right) d\left(y_{n}, q\right)+\gamma_{n} d\left(y_{n}, q\right) \\
& \leq d\left(y_{n}, q\right) \\
& \leq d\left(x_{n}, q\right) .
\end{aligned}
$$

This presents that $\left\{d\left(x_{n}, q\right)\right\}$ is bounded below and decreasing. Then, $\lim _{n \longrightarrow \infty} d\left(x_{n}, q\right)$ exists for each $q \in F(T)$.

Lemma 6. Let $D$ be a nonempty closed convex subset of $X$ and $T: D \longrightarrow D$ be a mapping which satisfies the condition $\left(C_{\lambda}\right)$ for some $\lambda \in(0,1)$ and the condition $(E)$. For an arbitrary chosen $x_{0} \in D$, a sequence $\left\{x_{n}\right\}$ is defined by (15). Then, $F(T) \neq \varnothing$ if and only if $\left\{x_{n}\right\}$ is bounded and $\lim _{n \longrightarrow \infty} d\left(x_{n}, T x_{n}\right)=0$.

Proof. Assume that $F(T) \neq \varnothing$, and let $q \in F(T)$. So, it follows from Lemma 5, $\left\{x_{n}\right\}$ is bounded. Next, we will indicate that $\lim _{n \longrightarrow \infty} d\left(x_{n}, T x_{n}\right)=0$. Since $T$ satisfies the condition $\left(C_{\lambda}\right)$ for some $\lambda \in(0,1)$ and $q \in F(T)$, for each $n \in \mathbb{N}$ we obtain that

$$
\lambda d(q, T q)=0 \leq d\left(q, x_{n}\right) .
$$

Therefore,

$$
d\left(T q, T x_{n}\right) \leq d\left(q, x_{n}\right)
$$

From Lemma 5, we achieve $\lim _{n \longrightarrow \infty} d\left(x_{n}, q\right)$ exists for all $q \in F(T)$. Assume that $\lim _{n \longrightarrow \infty} d\left(x_{n}, q\right)=s \geq 0$. If $s=0$, then

$$
\begin{aligned}
d\left(x_{n}, T x_{n}\right) & \leq d\left(x_{n}, q\right)+d\left(q, T x_{n}\right) \\
& \leq d\left(x_{n}, q\right)+d\left(T q, T x_{n}\right) \leq 2 d\left(x_{n}, q\right) .
\end{aligned}
$$

Letting limit as $n \longrightarrow \infty$ on both sides of the above estimate, we have

$$
\lim _{n \longrightarrow \infty} d\left(x_{n}, T x_{n}\right)=0 .
$$

If $s>0$, then

$$
\begin{aligned}
d\left(z_{n}, q\right) & =d\left(W\left(x_{n}, T x_{n}, \omega_{n}\right), q\right) \\
& \leq\left(1-\omega_{n}\right) d\left(x_{n}, q\right)+\omega_{n} d\left(T x_{n}, q\right) \\
& =\left(1-\omega_{n}\right) d\left(x_{n}, q\right)+\omega_{n} d\left(T x_{n}, T q\right) \\
& \leq\left(1-\omega_{n}\right) d\left(x_{n}, q\right)+\omega_{n} d\left(x_{n}, q\right) \leq d\left(x_{n}, q\right) .
\end{aligned}
$$

Taking lim sup as $n \longrightarrow \infty$ on the above inequality,

$$
\limsup _{n \longrightarrow \infty} d\left(z_{n}, q\right) \leq \limsup _{n \longrightarrow \infty} d\left(x_{n}, q\right)=s .
$$

It follows from (18) and (19) that $d\left(x_{n+1}, q\right) \leq d\left(z_{n}, q\right)$. By taking lim inf as $n \longrightarrow \infty$ on both sides, we obtain that

$$
s \leq \liminf _{n \longrightarrow \infty} d\left(x_{n+1}, q\right) \leq \liminf _{n \longrightarrow \infty} d\left(z_{n}, q\right) .
$$

The relations (25) and (26) imply that

$$
\lim _{n \rightarrow \infty} d\left(z_{n}, q\right)=s \text {. }
$$

Since $d\left(T x_{n}, q\right)=d\left(T x_{n}, T q\right) \leq d\left(x_{n}, q\right)$, we get that

$$
\limsup _{n \rightarrow \infty} d\left(T x_{n}, q\right) \leq \limsup _{n \longrightarrow \infty} d\left(x_{n}, q\right) \leq s .
$$

It follows from Lemma 2 through (27) and (28) that

$$
\lim _{n \rightarrow \infty} d\left(x_{n}, T x_{n}\right)=0 \text {. }
$$

Conversely, suppose that $\left\{x_{n}\right\}$ is bounded and $\lim _{n \rightarrow \infty} d\left(x_{n}, T x_{n}\right)=0$. Then, it follows from Lemma 3 that $T$ has a fixed point, so we have $F(T)$ which is nonempty.

We now establish the $\Delta$-convergence theorem for generalized nonexpansive mappings in hyperbolic spaces.

Theorem 1. Let $D$ be a nonempty closed convex subset of $X$ and $T: D \longrightarrow D$ be a mapping which satisfies the condition $\left(C_{\lambda}\right)$ for some $\lambda \in(0,1)$ and the condition $(E)$ with $F(T) \neq \varnothing$. Let $\left\{x_{n}\right\}$ be a sequence defined by (15). Then, $\left\{x_{n}\right\}$ $\Delta$-converges to a fixed point of $T$.

Proof. It follows from Lemma 6 that $\left\{x_{n}\right\}$ is a bounded sequence. Thus, $\left\{x_{n}\right\}$ has a $\Delta$-convergent subsequence. Now, we are going to show that every $\Delta$-convergent subsequence of $\left\{x_{n}\right\}$ has a unique $\Delta$-limit in $F(T)$. Let $w$ and $z$ be $\Delta$-limits of the subsequence $\left\{w_{n}\right\}$ and $\left\{z_{n}\right\}$ of $\left\{x_{n}\right\}$, respectively. From Lemma 1 , we have $A C\left(D,\left\{w_{n}\right\}\right)=\{w\}$ and $A C\left(D,\left\{z_{n}\right\}\right)=\{z\}$. By Lemma 6, we obtain that $\lim _{n \longrightarrow \infty} d\left(w_{n}, T w_{n}\right)=0$ and $\lim _{n \longrightarrow \infty} d\left(z_{n}, T z_{n}\right)=0$. Next, we prove that $w$ and $z$ are fixed points of $T$ and reunique should be are unique. Since $T$ satisfies the condition $(E)$, there exists $\mu_{w} \geq 1$ such that

$$
d\left(w_{n}, T w\right) \leq \mu_{w} d\left(w_{n}, T w_{n}\right)+d\left(w_{n}, w\right) .
$$

Letting lim sup as $n \longrightarrow \infty$ on both sides of the above inequality, we get

$$
\begin{aligned}
r_{a}\left(T w,\left\{w_{n}\right\}\right) & =\limsup _{n \longrightarrow \infty} d\left(w_{n}, T w\right) \\
& \leq \limsup _{n \longrightarrow \infty}\left[\mu_{w} d\left(w_{n}, T w_{n}\right)+d\left(w_{n}, w\right)\right] \\
& \leq \limsup _{n \longrightarrow \infty} d\left(w_{n}, w\right)=r_{a}\left(w,\left\{w_{n}\right\}\right) .
\end{aligned}
$$

The uniqueness of the asymptotic center implies $T w=w$. Thus, $w$ is a fixed point of $T$. Similarly, we also have $z$ as a fixed point of $T$. Lastly, we show that $w=z$. Suppose $w \neq z$, and so by the uniqueness of an asymptotic center, we have 


$$
\begin{aligned}
\limsup _{n \longrightarrow \infty} d\left(x_{n}, w\right)= & \limsup _{n \longrightarrow \infty} d\left(w_{n}, w\right) \\
& <\limsup _{n \longrightarrow \infty} d\left(w_{n}, z\right) \\
= & \limsup _{n \longrightarrow \infty} d\left(x_{n}, z\right) \\
= & \underset{n \longrightarrow \infty}{\limsup } d\left(z_{n}, z\right) \\
& <\limsup _{n \longrightarrow \infty} d\left(z_{n}, w\right) \\
= & \limsup _{n \longrightarrow \infty} d\left(x_{n}, w\right) .
\end{aligned}
$$

This is a contradiction. Thus, $w=z$. Then, $\left\{x_{n}\right\}$ $\Delta$-converges to a fixed point of $T$.

Next, we present some strong convergence theorems.

Theorem 2. Let $D$ be a nonempty closed convex subset of $X$ and $T: D \longrightarrow D$ be a mapping which satisfies the condition $\left(C_{\lambda}\right)$ for some $\lambda \in(0,1)$ and the condition $(E)$ with $F(T) \neq \varnothing$. Let $\left\{x_{n}\right\}$ be a sequence defined by (15). Then, $\left\{x_{n}\right\}$ converges strongly to a fixed point of $T$ if and only if $\liminf _{n \longrightarrow \infty} d\left(x_{n}, F(T)\right)=0$.

Proof. Assume that $\left\{x_{n}\right\}$ converges strongly to $q \in F(T)$. So, $\lim _{n \rightarrow \infty} d\left(x_{n}, q\right)=0$. Because $0 \leq d\left(x_{n}, F(T)\right) \leq d\left(x_{n}, q\right)$, therefore $\lim \inf _{n \rightarrow \infty} d\left(x_{n}, F(T)\right)=0$.

Conversely, suppose that $\lim _{\inf _{n \rightarrow \infty}} d\left(x_{n}, F(T)\right)=0$. It follows from Lemma 5 , and we get $d\left(x_{n+1}, q\right) \leq d\left(x_{n}, q\right)$ for all $q \in F(T)$. Thus, $d\left(x_{n+1}, F(T)\right) \leq d\left(x_{n}, F(T)\right)$. Therefore, $\lim _{n \longrightarrow \infty} d\left(x_{n}, F(T)\right)$ exists. From the assumption of our theorem, $\quad \liminf _{n \longrightarrow \infty} d\left(x_{n}, F(T)\right)=0$, so we have $\lim _{n \longrightarrow \infty} d\left(x_{n}, F(T)\right)=0$. Next, we prove that $\left\{x_{n}\right\}$ is a Cauchy sequence in $D$. Let for each $\varepsilon>0$. Since $\lim _{n \longrightarrow \infty} d\left(x_{n}, F(T)\right)=0$, for any given $\varepsilon>0$, there is $n_{0} \in \mathbb{N}$ such that

$$
d\left(x_{n}, F(T)\right)<\frac{\varepsilon}{2} \text { for all } n \geq n_{0} .
$$

In particular, $\inf \left\{d\left(x_{n_{0}}, q\right): q \in F(T)\right\}<(\varepsilon / 2)$. Then, there exists $q^{\prime} \in F(T)$ such that $d\left(x_{n_{0}}, q^{\prime}\right)<(\varepsilon / 2)$.

For any $m, n \geq n_{0}$, we get

$$
\begin{aligned}
d\left(x_{n+m}, x_{n}\right) & \leq d\left(x_{n+m}, q^{\prime}\right)+d\left(q^{\prime}, x_{n}\right) \\
& \leq d\left(x_{n_{0}}, q^{\prime}\right)+d\left(q^{\prime}, x_{n_{0}}\right) \\
& \leq \frac{\varepsilon}{2}+\frac{\varepsilon}{2}=\varepsilon .
\end{aligned}
$$

This shows that $\left\{x_{n}\right\}$ is a Cauchy sequence in $D$. Since $D$ is a closed subset of a complete hyperbolic space $X, D$ is complete. Then, $\left\{x_{n}\right\}$ must converge to a point in $D$. Let $\lim _{n \longrightarrow \infty} x_{n}=z \in D$. Now, we show that $z$ is a fixed point of $T$. Since $T$ satisfies the condition $(E)$, there exists $\mu \geq 1$ such that

$$
d\left(x_{n}, T z\right) \leq \mu d\left(x_{n}, T x_{n}\right)+d\left(x_{n}, z\right) .
$$

Letting $n \longrightarrow \infty$, it follows from Lemma 6 that $d\left(x_{n}, T x_{n}\right)=0$. We have $d(z, T z)=0$. Then, $z$ is a fixed point of $T$. Hence, $\left\{x_{n}\right\}$ converges strongly to a point in $F(T)$.

Theorem 3. Let $D$ be a nonempty closed convex subset of $X$ and $T: D \longrightarrow D$ be a mapping which satisfies the condition $\left(C_{\lambda}\right)$ for some $\lambda \in(0,1)$ and the condition $(E)$ with $F(T) \neq \varnothing$. If a sequence $\left\{x_{n}\right\}$ is determined by (15) and $T$ satisfies condition (I), then $\left\{x_{n}\right\}$ converges strongly to a fixed point of $T$.

Proof. From Lemma 5, we have $\lim _{n \longrightarrow \infty} d\left(x_{n}, q\right)$ exists for each $q \in F(T)$ and $\lim _{n \longrightarrow \infty} d\left(x_{n}, q\right)=s$ for some $s \geq 0$. Let $q \in F(T)$. If $s=0$, we obtain the desired result. Assume that $s>0$. From Lemma 6, we obtain that $\lim _{n \longrightarrow \infty} d\left(x_{n}, T x_{n}\right)=0$. Since $T$ satisfies condition $(I)$, we have

$$
\lim _{n \longrightarrow \infty} f\left(d\left(x_{n}, F(T)\right)\right) \leq \lim _{n \longrightarrow \infty} d\left(x_{n}, T x_{n}\right)=0 .
$$

Therefore,

$$
\lim _{n \longrightarrow \infty} f\left(d\left(x_{n}, F(T)\right)\right)=0 .
$$

From $f:[0, \infty) \longrightarrow[0, \infty)$ is a nondecreasing mapping satisfying $f(0)=0$ and $f(t)>0$ for each $t \in(0, \infty)$, we have $\lim _{n \rightarrow \infty} d\left(x_{n}, F(T)\right)=0$. By applying Theorem 2 , we obtain the desired result.

\section{Numerical Example}

First, we present an example of the convergence for a mapping satisfying the condition $\left(C_{\lambda}\right)$ and the condition $(E)$, but it fails to satisfy the condition $(C)$. Next, we give an example to compare the speed of convergence of our generalized $\mathrm{M}$-iteration process with $\mathrm{M}$-iteration process of Ullah et al. [6].

Example 2. Let $X=\mathbb{R}$ with metric $d(x, y)=|x-y|$ and $D=[0,1]$. Define $W: X^{2} \times[0,1] \longrightarrow X$ by $W(x, y, \gamma):=$ $\gamma x+(1-\gamma) y$ for each $x, y \in X$ and $\gamma \in[0,1]$. Thus, $(X, d, W)$ is a complete uniformly hyperbolic space with a monotone modulus of uniform convexity and $D$ is a nonempty compact convex subset of $X$. For a given $\lambda \in(0,1)$, let $T: D \longrightarrow D$ defined by

$$
T x= \begin{cases}\frac{x}{2}, & x \neq 1, \\ \frac{1+\lambda}{2+\lambda}, & x=1 .\end{cases}
$$

By [2], it is shown that the mapping $T$ satisfies the condition $\left(C_{\lambda}\right)$ but it fails to satisfy the condition $\left(C_{\lambda}^{\prime}\right)$ whenever $0<\lambda^{\prime}<\lambda$. Moreover, $T$ satisfies the condition $(E)$ for some $\mu=(2+\lambda) / 2$. For an initial point $x_{0}=0.1$ and $\omega_{n}=n /(10 n+2), \sigma_{n}=1 /(\sqrt{3 n+7})$, and $\gamma_{n}=1 /(\sqrt{3 n+7})$, we have $\left\{\omega_{n}\right\},\left\{\sigma_{n}\right\}$, and and $\left\{\gamma_{n}\right\}$ belonging in $[0,1]$. Set the stop parameter to $\left|x_{n}-0\right| \leq 10^{-15}$, where 0 is a fixed point of $T$. By using MATLAB, we compute the convergence of the 
generalized M-iteration (15). The numerical experiments of all iterations for approximating the fixed point 0 are given in Table 1, and the convergence of an iterative sequence generated by generalized M-iteration process is shown in Figure 1.

Example 3. Let a mapping $T:[0,1] \longrightarrow[0,1]$ be defined by

$$
T x= \begin{cases}1-x, & x \in\left[0, \frac{1}{8}\right), \\ \frac{x+7}{8}, & x \in\left[\frac{1}{8}, 1\right] .\end{cases}
$$

By the proof of [6], it is shown that a mapping $T$ satisfies the condition $(C)$. Then, $T$ also satisfies the condition $\left(C_{\lambda}\right)$ for some $\lambda \in(0,1)$ and the condition $(E)$ for some $\mu=3$. For an initial value $x_{0}=0.9$ and $\omega_{n}=n /(10 n+2), \sigma_{n}=1$, $\gamma_{n}=1$, and $\left\{\omega_{n}\right\},\left\{\sigma_{n}\right\}$, and $\left\{\gamma_{n}\right\}$ belong in $[0,1]$. Set the stop parameter to $\left|x_{n}-1\right| \leq 10^{-15}$, where 1 is a fixed point of $T$. We compare our iteration process (15) with M-iteration process (4). Numerically, we can easily see that the sequence generated by the generalized $\mathrm{M}$-iteration converges faster than the sequence generated by the $\mathrm{M}$-iteration process as given in Table 2 and Figure 2.

\section{Applications to Integral Equations}

In this section, we prove the existence of a solution of a nonlinear quadratic integral equation taken from Allahari et al. [19].

Let $C(I)$ be the set of all continuous functions defined on $I=[0,1]$ and $d: C(I) \times C(I) \longrightarrow \mathbb{R}$ defined by

$$
d(x, y)=\sup _{t \in I}|x(t)-y(t)| \text {, for all } x, y \in C(I) \text {. }
$$

We can see that $(C(I), d)$ is a metric space and a hyperbolic space with the modulus of uniform convexity.

Let $\Gamma$ be the set of functions $\gamma:[0,+\infty) \longrightarrow[0,+\infty)$ which satisfy the following condition:

(i) $\gamma$ is nondecreasing and $\gamma(t) \leq t$ for all $t \in[0,+\infty)$.

Consider the nonlinear quadratic equation as follows:

$$
x(t)=h(t)+\omega \int_{0}^{1} k(t, s) f(s, x(s)) \mathrm{d} s, t \in I, \omega \geq 0,
$$

where $h: I \longrightarrow \mathbb{R}, f: I \times \mathbb{R} \longrightarrow \mathbb{R}$, and $k: I \times I \longrightarrow \mathbb{R}$. From now on, let $T: C(I) \longrightarrow C(I)$ be defined by

$$
T(x)(t)=h(t)+\omega \int_{0}^{1} k(t, s) f(s, x(s)) \mathrm{d} s \text { for } t \in I .
$$

Suppose that the following conditions hold:

(A1) $h: I \longrightarrow \mathbb{R}$ is continuous;

(A2) $f: I \times \mathbb{R} \longrightarrow \mathbb{R}$ is continuous, $f(t, x) \geq 0$ and there exist $L \geq 0$ and $\gamma \in \Gamma$ such that for all $t \in I$ and for all $a, b \in \mathbb{R},|f(t, a)-f(t, b)| \leq L \gamma(|a-b|)$;

(A3) $k: I \times I \longrightarrow \mathbb{R}$ is continuous at $t \in I$ for every $s \in I$ and measurable at $s \in I$ for all $t \in I$ such that $k(t, s) \geq 0$ and $\int_{0}^{1} k(t, s) \mathrm{d} s \leq K$;
TABLE 1: Sequence generated by generalized M-iteration process.

\begin{tabular}{cc}
\hline Iterate & Generalized M-iteration process \\
\hline$x_{0}$ & 0.1 \\
$x_{1}$ & 0.032887490635253 \\
$x_{2}$ & 0.011717192936408 \\
$x_{3}$ & 0.004203808068737 \\
$x_{4}$ & 0.001512138889767 \\
$\vdots$ & $\vdots$ \\
$x_{29}$ & 0.000000000000013 \\
$x_{30}$ & 0.000000000000005 \\
$x_{31}$ & 0.000000000000002 \\
$x_{32}$ & 0.000000000000000 \\
\hline
\end{tabular}

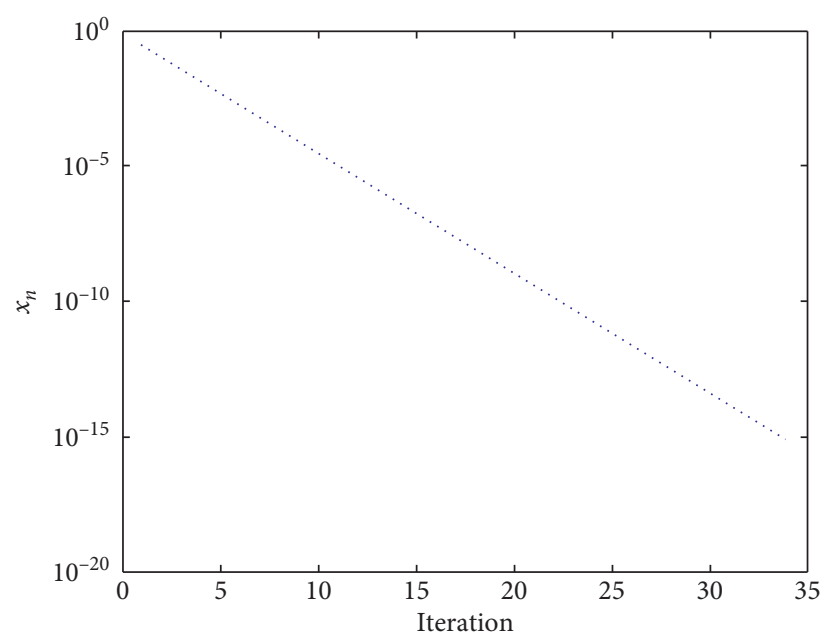

FIgURE 1: Convergence of an iterative sequence generated by generalized $\mathrm{M}$-iteration process.

TABLE 2: Sequences generated by generalized M-iteration process and M-iteration process.

\begin{tabular}{lcc}
\hline Iterate & Generalized M-iteration process & M-iteration process \\
\hline$x_{0}$ & 0.9 & 0.9 \\
$x_{1}$ & 0.9998046875 & 0.9984375 \\
$x_{2}$ & 0.999999396006266 & 0.999977366129557 \\
$x_{3}$ & 0.999999998069622 & 0.999999674477361 \\
$x_{4}$ & 0.999999999993755 & 0.999999995330944 \\
$x_{5}$ & 0.999999999999980 & 0.999999999933126 \\
$x_{6}$ & 1 & 0.999999999999043 \\
$x_{7}$ & 1 & 0.999999999999986 \\
$x_{8}$ & 1 & 1 \\
$x_{9}$ & 1 & 1 \\
$x_{10}$ & 1 & 1 \\
\hline
\end{tabular}

(A4) $\omega K L \leq 1$;

(A5) The sequence $\left\{x_{n}\right\}$ defined as in (15) is bounded and $\lim _{n \longrightarrow \infty} d\left(x_{n}, T x_{n}\right)=0$.

Theorem 4. Under assumptions (A1)-(A5), the integral equation (41) has a solution in $C(I)$.

Proof. Let $x, y \in C(I)$ such that

$$
\lambda d(x, T x) \leq d(x, y),
$$




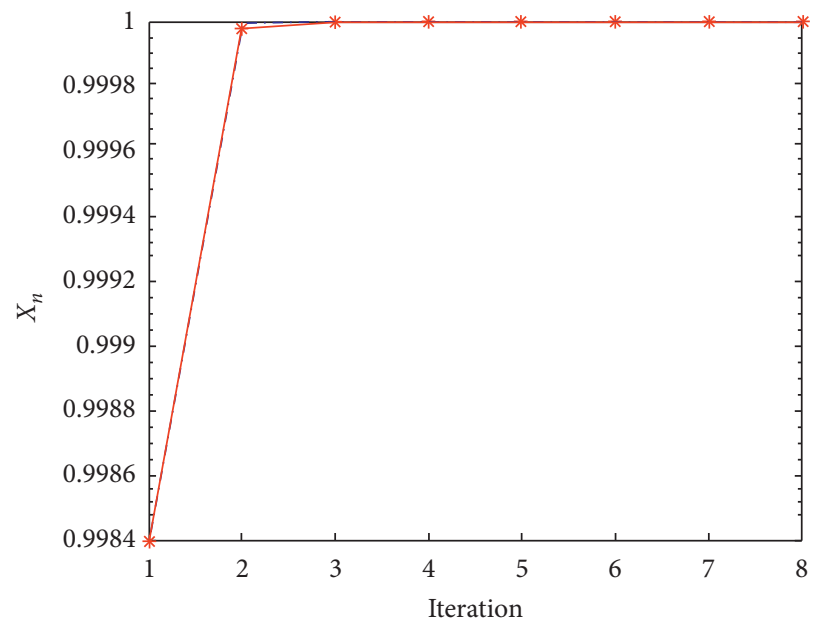

FiguRE 2: Convergence of iterative sequences generated by generalized M-iteration process and M-iteration process to the fixed point 1 of a mapping $T$ defined in Example 3.

for some $\lambda \in(0,1)$. Using (A2), we obtain that

$$
\begin{aligned}
|T(x)(t)-T(y)(t)|= & \mid h(t)+\omega \int_{0}^{1} k(t, s) f(s, x(s)) \mathrm{d} s-h(t) \\
& -\omega \int_{0}^{1} k(t, s) f(s, y(s)) \mathrm{d} s \mid \\
\leq & \omega \int_{0}^{1} k(t, s)|f(s, x(s))-f(s, y(s))| \mathrm{d} s \\
\leq & \omega \int_{0}^{1} k(t, s) L \gamma(|x(s)-y(s)|) \mathrm{d} s .
\end{aligned}
$$

Since $\gamma$ is nondecreasing, we have

$$
\gamma(|x(s)-y(s)|) \leq \gamma\left(\sup _{s \in I}|x(s)-y(s)|\right)=\gamma(d(x, y)) \text {. }
$$

From (A3), we obtain that

$$
|T(x)(t)-T(y)(t)| \leq \omega K L \gamma(d(x, y)) .
$$

Therefore,

$$
\begin{aligned}
d(T x, T y) & =\sup _{t \in I}|T(x)(t)-T(y)(t)| \\
& \leq \omega K \operatorname{LL}(d(x, y)) \\
& \leq d(x, y) .
\end{aligned}
$$

This implies that $T$ satisfies the condition $\left(C_{\lambda}\right)$ for $\lambda \in(0,1)$.

Let $x, y \in C(I)$. We obtain that

$$
\begin{aligned}
|x(t)-T(y)(t)|= & \left|x(t)-\left(h(t)+\omega \int_{0}^{1} k(t, s) f(s, y(s)) \mathrm{d} s\right)\right| \\
& \leq\left|x(t)-\left(h(t)+\omega \int_{0}^{1} k(t, s) f(s, x(s)) \mathrm{d} s\right)\right| \\
& +\left|\left(h(t)+\omega \int_{0}^{1} k(t, s) f(s, x(s)) \mathrm{d} s\right)-\left(h(t)+\omega \int_{0}^{1} k(t, s) f(s, y(s)) \mathrm{d} s\right)\right| \\
= & |x(t)-T(x)(t)|+|T(x)(t)-T(y)(t)| .
\end{aligned}
$$

This implies that

$$
\begin{aligned}
d(x, T y) & \leq d(x, T x)+d(T x, T y) \\
& \leq d(x, T x)+d(x, y) \\
& \leq \mu d(x, T x)+d(x, y)
\end{aligned}
$$

for some $\mu \geq 1$. Then, $T$ satisfies the condition $(E)$. By Lemma 3 and (A5), we get that $F(T) \neq \varnothing$. Therefore, all assumptions in Theorem 1 are satisfied. Hence, $\left\{x_{n}\right\}$ $\Delta$-converges to a fixed point of $T$ in $C(I)$. This implies that the integral equation (41) has a solution in $C(I)$. 


\section{Conclusion Remark}

Our space is a uniformly convex hyperbolic space which is a natural generalization of a uniformly convex Banach space. Since our mappings are more general than mappings of Ullah et al. [6] and the sequence generated by the generalized $\mathrm{M}$-iteration process (15) converges faster than the sequence generated by the $\mathrm{M}$-iteration process (4), our results improve the results of Ullah et al. [6].

\section{Data Availability}

No data were used to support this study.

\section{Conflicts of Interest}

The authors declare that there are no conflicts of interest.

\section{Acknowledgments}

The first author was supported by the Science Achievement Scholarship of Thailand (SAST). The third author would like to express her deep thanks to Naresuan University for supporting this research under grant R2563C002.

\section{References}

[1] T. Suzuki, "Fixed point theorems and convergence theorems for some generalized nonexpansive mappings," Journal of Mathematical Analysis and Applications, vol. 340, pp. 10881095, 2008.

[2] J. García-Falset, E. Llorens-Fuster, and T. Suzuki, "Fixed point theory for a class of generalized nonexpansive mappings," Journal of Mathematical Analysis and Applications, vol. 375, pp. 185-195, 2011.

[3] W. R. Mann, "Mean value methods in iteration," Proceedings of American Mathematical Society, vol. 4, pp. 506-510, 1953.

[4] R. P. Agarwal, D. O'Regan, and D. R. Sahu, "Iterative construction of fixed points of nearly asymptotically nonexpansive mappings," Nonlinear Convex A, vol. 8, pp. 61-79, 2007.

[5] F. Gursoy and V. Karakaya, A Picard-S Hybrid Type Iteration Method for Solving a Differential Equation with Retarded Argument, 2014, http://arxiv.org/abs/1403.2546v2.

[6] K. Ullah and M. Arshad, "Numerical reckoning fixed points for Suzuki's generalized nonexpansive mappings via new oteration process," Filomat, vol. 32, no. 1, pp. 187-196, 2018.

[7] U. Kohlenbach, "Some logical metatheorems with applications in functional analysis," Transactions of the American Mathematical Society, vol. 357, no. 1, pp. 89-129, 2005.

[8] S. Reich and I. Shafrir, "Nonexpansive iterations in hyperbolic spaces," Nonlinear Analysis: Theory, Methods \& Application, vol. 15 , no. 6 , pp. 537-558, 1990.

[9] N. Bridson and A. Haefliger, Metric Spaces of Non-positive Curvature, Springer, Berlin, Germany, 1999.

[10] L. Leustean, "A quadratic rate of asymptotic regularity for CAT(0) spaces," Journal of Mathematical Analysis and Applications, vol. 235, pp. 386-399, 2007.

[11] J. Markin, "Continuous depence of fixed point sets," Proceedings of American Mathematical Society, vol. 38, pp. 545$547,1973$.

[12] J. Zhou and Y. Cui, "Fixed point properties of Suzuki generalized nonexpansive set-valued mappings in complete
CAT(0) spaces," Journal of Computational Analysis and Applications, vol. 24, no. 1, pp. 91-104, 2018.

[13] S. Dhompongsa, W. A. Kirk, and B. Panyanak, "Nonexpansive set-valued mappings in metric and Banach spaces," Nonlinear Convex A, vol. 8, pp. 35-45, 2007.

[14] H. F. Senter and W. G. Dotson, "Approximating fixed points of nonexpansive mappings," Proceedings of American Mathematical Society, vol. 44, pp. 375-380, 1974.

[15] S. Dhompongsa and B. Panyanak, "On $\Delta$-convergence theorem in CAT(0) spaces," Computers \& Mathematics with Applications, vol. 56, no. 10, pp. 2572-2579, 2008.

[16] L. Leustean, "Nonexpansive iteration in uniformly convex $W$-hyperbolic spaces," in Nonlinear Analysis and Optimization I. Nonlinear Analysis. Contemporary Mathematics, A. Leizarowitz, B. S. Mordukhovich, I. Shafrir, and A. Zaslavski, Eds., Ramat Gan American Mathematical Society, Bar Ilan University, Ramat Gan, Israel, 2010.

[17] A. R. Khan, H. Fukhar-ud-din, and M. A. A. Kuan, "An implichit algorithm for two finite families of nonexpansive maps in hyperbolic spaces," Fixed Point Theory A, vol. 2012, no. 54, 2012.

[18] M. Imdad and S. Dashputre, "Fixed point approximation of Picard normal S-iteration process for generalized nonexpansive mappings in hyperbolic spaces," Mathematical Sciences, vol. 10, no. 3, pp. 131-138, 2016.

[19] R. Allahyari, R. Arab, and A. S. Haghighi, "Fixed point of admissible almost contractive type mappings on $b$-metric spaces with an applications to quadratic integral equations," Journal of Inequalities and Applications, vol. 2015, p. 32, 2015. 